\title{
ATIVIDADE INSETICIDA DE ÓLEOS ESSENCIAIS DE Pelargonium graveolens l'Herit E Lippia alba (Mill) N. E. Brown SOBRE Spodoptera frugiperda (J. E. Smith)
}

Edenilson dos S. Niculau, Péricles B. Alves, Paulo Cesar de L. Nogueira e Valéria Regina de S. Moraes

Departamento de Química, Centro de Ciências Exatas e Tecnologia, Universidade Federal de Sergipe, Av. Marechal Rondon S/N, 49100-000 São Cristóvão - SE, Brasil

Andréia P. Matos, Antonio R. Bernardo, Ana C. Volante, João B. Fernandes*, Maria F. G. F. da Silva e Arlene G. Corrêa Departamento de Química, Centro de Ciências Exatas e Tecnologia, Universidade Federal de São Carlos, Rod. Washington Luís, Km 235, 13.565-905 São Carlos - SP, Brasil

Arie F. Blank e Anderson de C. Silva

Departamento de Engenharia Agronômica, Universidade Federal de Sergipe, Av. Marechal Rondon S/N, 49100-000 São Cristóvão - SE, Brasil

Leandro do P. Ribeiro

Departamento de Entomologia e Acaralogia, Escola Superior de Agricultura "Luiz de Queiroz", Universidade de São Paulo, Av. Pádua Dias, 11, 13418-900 Piracicaba - SP, Brasil

Recebido em 7/6/13; aceito em 14/6/13; publicado na web em 2/8/13

INSECTICIDAL ACTIVITY OF ESSENTIAL OILS OF Pelargonium graveolens l'Herit AND Lippia alba (Mill) N. E. Brown AGAINST Spodoptera frugiperda (J. E. Smith). Insecticidal activity of essential oils of Pelargonium graveolens, Lippia alba and compounds geraniol, linalool, 1,8-cineole, limonene, carvone, citral and Azamax ${ }^{\circledR}$ were evaluated against Spodoptera frugiperda. Topical application assay showed essential oil of P. graveolens has acute toxicity against Spodoptera frugiperda larvae (third instar) with $\mathrm{LD}_{50} 1.13 \mu \mathrm{g} / \mathrm{mg}$ per insect and $\mathrm{LD}_{90} 2.56 \mu \mathrm{g} / \mathrm{mg}$ per insect. Three essential oils of $L$. alba also exhibited insecticidal activity with $\mathrm{LD}_{50}$ ranging from 1.20 to $1.56 \mu \mathrm{g} / \mathrm{mg}$ per insect and $\mathrm{LD}_{90}$ from 2.60 to $3.75 \mu \mathrm{g} / \mathrm{mg}$ per insect. Geraniol, linalool, carvone and citral caused significant mortality of 30, 90, 84 and 64\% respectively, compared to negative control. The bioinsecticide, Azamax ${ }^{\circledR}$, caused lower mortality than the compounds of the essential oils.

Keywords: Lippia alba; Pelargonium graveolens; Spodoptera frugiperda.

\section{INTRODUÇÃO}

A lagarta-do-cartucho do milho, Spodoptera frugiperda (J. E. Smith), é uma das principais pragas da cultura do milho, podendo seu dano levar à redução de até $34 \%$ no rendimento de grãos, dependendo principalmente do estádio da cultura em que ocorre o ataque. ${ }^{1,2}$ No milho, a lagarta é usualmente controlada com inseticidas sintéticos empregados quando a desfolhação é notada nas plantações. No entanto, há diversos problemas associados ao uso de pesticidas, especialmente na seleção de populações resistentes a tais moléculas e os efeitos maléficos sobre insetos benéficos (entomófagos) que auxiliam no equilíbrio ecológico. ${ }^{2}$ Dessa forma, novas técnicas alternativas de controle vêm sendo buscadas, incluindo-se, dentre elas, os inseticidas de origem vegetal. A busca de novos derivados vegetais tem sido intensificada uma vez que, de forma geral, os inseticidas naturais não são persistentes, ou seja, degradam-se com maior velocidade que os sintéticos, não deixando resíduos nos alimentos ou no meio-ambiente.

Os terpenos encontrados em óleos essenciais tais como monoterpenos e sesquiterpenos, compostos produzidos no metabolismo secundários das plantas, são alvos de inúmeros estudos como bioinseticidas, pois uma das principais finalidades destas classes de compostos é a proteção contra insetos-pragas em plantas. ${ }^{3}$

Lippia alba (Mill) N. E. Brown e Pelargonium graveolens L'Herit são grandes produtoras de óleos essenciais e tem diversas aplicações, principalmente nas indústrias de perfumaria, cosméticos e farmacêutica. L. alba é uma planta medicinal aromática que ocorre praticamente em todas as regiões do Brasil. ${ }^{4}$ Vários compostos

*e-mail: djbf@power.ufscar.br voláteis fazem parte da composição química majoritária do óleo desta planta, entre os quais se destacam citral (geranial + neral), linalol, limoneno, carvona, cânfora e 1,8-cineol. ${ }^{5}$ P. graveolens é conhecida como malva cheirosa ou gerânio e foi introduzida na Índia no início do século $20 .{ }^{6}$ Segundo alguns estudos, citronelol, geraniol, linalol e formiato de citronelila são os principais componentes presentes no óleo essencial desta espécie. ${ }^{7}$

A atividade repelente e inseticida de vários óleos essenciais e terpenos como 1,8-cineol, $\alpha$-terpineol, timol, citronelal e citronelol, $\alpha-$ e $\beta$-pineno, mirceno, linalol, carvacrol, limoneno, mentol, entre outros, foram descritas para diversos insetos. ${ }^{8}$ Entre os óleos essenciais já estudados, o de citronela (Cymbogon winterianus J.), o de mil-folhas (Achiellea millefolium L.) e o de tomilho (Thymus vulgaris L.) já demonstraram atividade inseticida e repelente, atratividade ou deterrência alimentar sobre a lagarta $S$. frugiperda, ${ }^{9}$ porém, pelo nosso conhecimento, não há relatos sobre a ação inseticida do óleo essencial de $L$. alba e $P$. graveolens, bem como desconhecemos o estudo sobre a aplicação de seus principais compostos majoritários sobre à lagarta do cartucho-do-milho.

Dessa forma, este trabalho teve como objetivo avaliar o efeito inseticida dos óleos essenciais de P. graveolens e L. alba e dos seus compostos terpênicos majoritários sobre à lagarta do cartucho-do-milho por meio de ensaio toxicológico (aplicação tópica).

\section{PARTE EXPERIMENTAL}

\section{Material vegetal}

P. graveolens, acesso PEL001, e L. alba foram cultivadas e 
coletadas na Fazenda Experimental Campus Rural da Universidade Federal de Sergipe (UFS), situado no município de São Cristóvão - SE, Brasil (latitude $10^{\circ} 55^{\prime} 26^{\prime \prime} \mathrm{S}$; longitude $37^{\circ} 11^{\prime} 53^{\prime \prime} \mathrm{W}$ ). As exsicatas das plantas estão depositada no herbário da UFS sob registro ASE-14844 para $P$. graveolens (PEL-001) e ASE-13495, ASE-13476, ASE-13469 para L. alba, LA-10 (quimiotipo neral - geranial), LA22 (quimiotipo linalol - 1,8-cineol) e LA-57 (quimiotipo limoneno - carvona), respectivamente.

\section{Extração dos óleos essenciais}

O óleo essencial de $P$. graveolens (PEL-001) foi extraído a partir de $200 \mathrm{~g}$ de folhas frescas submetidas à hidrodestilação em aparelho tipo Clevenger por 3 h. O óleo foi separado da solução aquosa (hidrolato) por decantação, secado com $\mathrm{Na}_{2} \mathrm{SO}_{4}$ anidro, transferido para frasco de vidro âmbar e estocado em freezer até análise por CG-EM e CG-DIC.

Os óleos essenciais de L. alba (LA-10, LA-22 e LA-57) foram extraídos a partir de $75 \mathrm{~g}$ de folhas secas em estufa com circulação de ar Marconi (MA-037/18) a $39 \pm 1{ }^{\circ} \mathrm{C}$ por cinco dias e submetidas ao mesmo tratamento acima.

\section{Ensaio de aplicação tópica sobre $S$. frugiperda}

Os ensaios de aplicações tópicas sobre S. frugiperda foram realizados no laboratório de Bioensaios do Departamento de Química da Universidade Federal de São Carlos (UFSCar) baseado na metodologia descrita por Paula et al. (2000). ${ }^{10}$ A criação de $S$. frugiperda foi mantida em laboratório a $25 \pm 1{ }^{\circ} \mathrm{C}$, U.R de $70 \pm 5 \%$ e fotofase de $12 \mathrm{~h}$, sendo as lagartas alimentadas com dieta artificial à base de feijão e gérmen de trigo e os adultos com solução de mel a $10 \% .^{11}$

Para realização dos bioensaios, foram preparadas soluções dos óleos essenciais em acetona em quatro diferentes concentrações $(24,48,96$ e $192 \mu \mathrm{g} / \mu \mathrm{L})$, sendo estas definidas em testes preliminares visando obter faixas de respostas, ou seja, intervalos de doses que ocasionassem mortalidades desde próximo de zero até próximo de $100 \%$, conforme método descrito por Finney. ${ }^{12}$ Feito isso, foram aplicadas topicamente $1 \mu \mathrm{L}$ das respectivas soluções (correspondendo as doses de 24, 48, 96 e $192 \mu \mathrm{g}$ de óleo essencial por lagarta) na região protorácica com o auxílio de uma microseringa (HAMILTON). O controle foi realizado sob as mesmas condições: $1 \mu \mathrm{L}$ de acetona aplicado em cada inseto. Para evitar possível morte do inseto por inanição, em cada grupo de larvas foram colocadas pequenas porções $(300,00 \mathrm{mg})$ da dieta artificial. Decorridos 24 horas da aplicação, avaliou-se a toxicidade aguda dos óleos essenciais por meio da contagem do número de lagartas mortas. Para cada nível dos tratamentos, foram realizadas 5 repetições, sendo expostas 10 lagartas de terceiro instar em cada repetição. As estimativas das $\mathrm{DL}_{50}$ e $\mathrm{DL}_{90}$ (doses necessárias para matar 50 e $90 \%$ da população de lagartas, respectivamente) foram expressas em $\mu \mathrm{g}$ de óleo essencial/ mg de inseto, ajustadas de acordo com a concentração da solução utilizada e o peso médio das lagartas expostas em cada nível de tratamento, obtidas mediante pesagem individual de cada inseto no momento da montagem do bioensaio. ${ }^{13}$

Para avaliação da atividade inseticida dos compostos geraniol, linalol, 1,8-cineol, limoneno, carvona e citral, utilizou-se uma dose discriminante de $3 \mu \mathrm{g}$ de composto/mg de inseto, sendo esta definida com base nas $\mathrm{DL}_{90}$ estimadas para os respectivos óleos essenciais. $\mathrm{O}$ bioinseticida natural Azamax ${ }^{\circledR}$ também foi avaliado na mesma dose dos compostos mencionados acima. Os demais procedimentos experimentais utilizados foram os mesmos descritos para o bioensaio com os óleos essenciais.

\section{Análise estatística}

As estimativas das doses letais dos óleos essenciais foram realizadas por meio de análise de Probit, ${ }^{12}$ utilizando o programa Poloplus 1.0.14

Modelos lineares generalizados ${ }^{15}$ do tipo quase-binomial foram usados para análise dos dados de proporções de mortalidade obtidos no bioensaio com os compostos majoritários dos óleos essenciais. A verificação da qualidade do ajuste foi feita por meio do uso do gráfico meio-normal de probabilidades com envelope de simulação. ${ }^{16}$ Quando houve diferença significativa entre os tratamentos, múltiplas comparações (teste de Tukey, $\mathrm{p}<0,05$ ) foram realizadas por meio da função glht do pacote multicomp com ajuste dos valores de $p$. Todas as análises foram realizadas utilizando-se o software estatístico "R", versão $2.15 .1 .^{17}$

\section{Cromatografia gasosa acoplada à espectrometria de massas (CG-EM) e cromatografia gasosa com detector de ionização por chamas (CG-DIC)}

As análises foram realizadas em cromatógrafo a gás (Shimadzu Corporation, Kyoto, Japan), hifenizado a um espectrômetro de massas (CG-EM) equipado com um autoinjetor Shimadzu modelo AOC-20i. As seguintes condições foram usadas: coluna capilar de sílica fundida J\&W Scientific DB-5MS (5\% fenilmetilpolissiloxano) com $30 \mathrm{~m} \mathrm{x}$ $0,2 \mathrm{~mm}$ d. i. $\mathrm{x} 0,25 \mu \mathrm{m}$ de espessura do filme, fluxo de $1 \mathrm{~mL} \mathrm{\textrm {min } ^ { - 1 }}$ de hélio como gás de arraste $(99,999 \%)$; volume de injeção $0,5 \mu \mathrm{L}$ (diluído em hexano); razão do split de 1:20; temperatura do injetor de $250{ }^{\circ} \mathrm{C}$; temperatura do detector $280{ }^{\circ} \mathrm{C}$. A temperatura foi programada para $50{ }^{\circ} \mathrm{C}$ por $1,5 \mathrm{~min}$, seguido de $4{ }^{\circ} \mathrm{C} \min ^{-1}$ até $200{ }^{\circ} \mathrm{C}$, então $10^{\circ} \mathrm{C}$ até $300{ }^{\circ} \mathrm{C}$, finalizando com uma isoterma de $10 \mathrm{~min}$ a $250{ }^{\circ} \mathrm{C}$. O espectro de massas foi conduzido a $70 \mathrm{eV}$ com varredura rápido de 0,85 scan s $^{-1}$ na faixa de massas de 40 a $550 \mathrm{Da}$.

As análises quantitativas dos constituintes químicos foram realizadas usando um cromatógrafo a gás com detector de ionização por chama (CG-DIC) Shimadzu CG-17A. Uma coluna capilar de sílica fundida ZB-5 (5\% fenil, 95\% dimetilpolissiloxano), $30 \mathrm{~m} \mathrm{x} \mathrm{0,25} \mathrm{mm}$ d.i., 0,25 $\mu \mathrm{m}$ de espessura do filme) foi empregada (Phenomenex, Torrance, CA, USA), sob as mesmas condições procedidas no CGEM. A quantificação de cada constituinte foi estimada pela normalização da área (\%). As concentrações dos compostos foram calculadas através da área dos picos no CG e organizadas em ordem de eluição.

\section{Identificação dos constituintes voláteis}

Os componentes voláteis foram identificados através da comparação de seu espectro de massas com espectros existentes na literatura, ${ }^{18}$ com espectros do banco de dados (WILEY8, NIST05, NIST21 e NIST107) do equipamento e, também, pela comparação dos índices de retenção com aqueles da literatura. Os indices de retenção $\left(I_{R}\right)$ foram determinados utilizando uma série homologa de n-alcanos $\left(\mathrm{C}_{9} \mathrm{H}_{20}-\mathrm{C}_{20} \mathrm{H}_{42}\right)$ injetados nas mesmas condições cromatográficas das amostras, utilizando a equação de Van den Dool e Kratz. ${ }^{19}$

\section{RESULTADOS E DISCUSSÃO}

Com base nos resultados obtidos, foi constatado que os óleos essenciais de $P$. graveolens (PEL-001) e L. alba (acessos LA-10, LA-22, LA-57) apresentam toxicidade aguda para lagartas de terceiro instar de $S$. frugiperda, via aplicação tópica, com dose letal média $\left(\mathrm{DL}_{50}\right)$ de 1,$13 ; 1,24 ; 1,56$ e $1,20 \mu \mathrm{g} / \mathrm{mg}$ de inseto e $\mathrm{DL}_{90}$ de 2,56; 2,60; 3,75 e 3,08 $\mu \mathrm{g} / \mathrm{mg}$ de inseto, respectivamente (Tabela 1). Porém, com base na comparação dos intervalos de confiança obtidos 
Tabela 1. Estimativas das $\mathrm{DL}_{50}$ e $\mathrm{DL}_{90}$ (em $\mu \mathrm{g} / \mathrm{mg}$ de inseto) e intervalo de confiança de óleos essenciais para lagartas terceiro instar de Spodopetra frugiperda, após 24 horas da aplicação tópica. Temp.: $25 \pm 1{ }^{\circ} \mathrm{C}$; U.R.: $70 \pm 5 \%$; fotofase: $12 \mathrm{~h}$

\begin{tabular}{|c|c|c|c|c|c|}
\hline Óleos essenciais & Coeficiente angular ( \pm EP) & $\mathrm{DL}_{50}(\mathrm{IC})^{\mathrm{a}}$ & $\mathrm{DL}_{90}(\mathrm{IC})^{\mathrm{a}}$ & $\chi^{2(\mathrm{~b})}$ & g.1.c \\
\hline Pelargonium graveolens (PEL-001) & $3,61 \pm 1,04$ & $1,13(0,83-1,45)$ & $2,56(1,83-7,23)$ & 1,09 & 2 \\
\hline Lippia alba (LA-10) & $3,98 \pm 0,95$ & $1,24(0,89-1,52)$ & $2,60(2,04-4,45)$ & 0,85 & 2 \\
\hline Lippia alba (LA-22) & $3,37 \pm 0,69$ & $1,56(1,18-2,02)$ & $3,75(2,75-6,99)$ & 1,21 & 2 \\
\hline Lippia alba (LA-57) & $3,13 \pm 0,74$ & $1,20(0,90-1,57)$ & $3,08(2,15-7,18)$ & 1,12 & 2 \\
\hline
\end{tabular}

${ }^{a}$ IC: intervalo de confiança a $95 \%$ de probabilidade de erro. ${ }^{(b)} \chi^{2}$ : valor de qui-quadrado calculado. ${ }^{\mathrm{c}}$ g.1.: graus de liberdade.

( $\mathrm{p} \leq 0,05$ ), foi verificado que os óleos essenciais estudados não diferiram entre si para nenhuma das doses estimadas $\left(\mathrm{DL}_{50}\right.$ e $\left.\mathrm{DL}_{90}\right)$. Além disso, todas as curvas de dose-resposta estimadas apresentaram coeficientes angulares (inclinação da curva) muito próximos, o que indica que lagartas de $S$. frugiperda respondem de forma semelhante aos diferentes óleos essenciais estudados. Valores altos de inclinação da curva indicam que pequenas variações na dose do óleo essencial promovem grandes variações na mortalidade, resultando em resposta homogênea da população. ${ }^{20}$

Os principais compostos majoritários dos óleos essenciais bem como um bioinseticida padrão $\left(\right.$ Azamax $\left.^{\circledR}\right)$ registrado no Brasil também foram avaliados sobre lagartas de $S$. frugiperda na dose de $3 \mu \mathrm{g} / \mathrm{mg}$ de inseto, via aplicação tópica. Com base nos resultados obtidos no bioensaio, foi constatado que os compostos geraniol, linalol, carvona e citral ocasionam mortalidades de 30, 90, 84 e 64\%, respectivamente, bem maiores do que o percentual de mortalidade provocada por Azamax ${ }^{\circledR}$ (Tabela 2).

Tabela 2. Mortalidade (médias \pm erro padrão) de lagartas de terceiro instar de Spodoptera frugiperda após 24 da aplicação tópica de diferentes compostos terpênicos e do bioinseticida Azamax ${ }^{\otimes}\left(3 \mu \mathrm{g} / \mathrm{mg}\right.$ de inseto). Temp: $25 \pm 1^{\circ} \mathrm{C}$, U.R.: $70 \pm 5 \%$; fotofase: $12 \mathrm{~h}$

\begin{tabular}{lc}
\hline Compostos & Mortalidade $(\%)^{*^{*}}$ \\
\hline Geraniol & $30,0 \pm 8,84 \mathrm{bc}$ \\
Linalol & $90,0 \pm 3,16 \mathrm{a}$ \\
1,8 -Cineol & $2,0 \pm 2,00 \mathrm{~d}$ \\
Limoneno & $4,0 \pm 2,44 \mathrm{~d}$ \\
Carvona & $84,0 \pm 5,09 \mathrm{a}$ \\
Citral & $64,00 \pm 7,07 \mathrm{ab}$ \\
Azamax & (azadiractina +3 -tigloilazadirachtol) \\
Controle negativo (acetona) & $14,00 \pm 5,09 \mathrm{~cd}$ \\
F & $3,0 \pm 2,00 \mathrm{~d}$ \\
Valor de $p$ & 26,96 \\
\hline
\end{tabular}

* Médias seguidas de letras distintas indicam diferenças significativas entre os tratamentos (GLM com distribuição quase-binomial seguido por teste post hoc de Tukey, $\mathrm{p}<0,05)$.

Com base em tais resultados, foi verificado que a alta toxicidade do óleo essencial de $P$. graveolens (PEL-001) deve estar associada à presença de altos percentuais de geraniol $(23,1 \%)$ (Tabela 3$)$, visto que este causou $30 \%$ de mortalidade (Tabela 2). No entanto, provavelmente outros compostos majoritários como o citronelol e linalol podem estar agindo sinergicamente para contribuir na ação tóxica frente às lagartas de $S$. frugiperda, fato ratificado na comparação da toxicidade do respectivo óleo essencial, expressa nas doses letais estimadas, com a mortalidade obtida no bioensaio onde o composto foi avaliado. Labinas et al. (2002) ${ }^{9}$ ao estudarem o óleo essencial de citronela (Cymbopogon winterianus J.), rico em citronelal e citronelol, demonstrou efeito inseticida e repelente para a referida espécie-praga.
Tabela 3. Composição química majoritária dos óleos essenciais de $P$. graveolens (PEL-001) e L. alba (LA-10, LA-22 e LA-57)

\begin{tabular}{lccccc}
\hline \multirow{2}{*}{ Compostos $^{\mathrm{a}}$} & $\mathrm{I}_{\mathrm{R}}{ }^{\mathrm{b}}$ & \multicolumn{4}{c}{ Área do pico (\%) } \\
\cline { 3 - 6 } & 1028 & $\mathrm{nd}^{\mathrm{d}}$ & $\mathrm{nd}$ & $\mathrm{nd}$ & 36,0 \\
\hline Limoneno & 1031 & $\mathrm{nd}$ & $\mathrm{nd}$ & 8,2 & $\mathrm{nd}$ \\
1,8-cineol & 1099 & 11,2 & 1,6 & 81,0 & $\mathrm{nd}$ \\
Linalol & 1226 & 27,6 & $\mathrm{nd}$ & $\mathrm{nd}$ & $\mathrm{nd}$ \\
Citronelol & 1238 & 0,7 & 32,9 & $\mathrm{nd}$ & $\mathrm{nd}$ \\
Neral & 1243 & $\mathrm{nd}$ & 0,7 & $\mathrm{nd}$ & 54,8 \\
Carvona & 1250 & 23,1 & $\mathrm{nd}$ & $\mathrm{nd}$ & $\mathrm{nd}$ \\
Geraniol & 1267 & 1,3 & 47,0 & $\mathrm{nd}$ & $\mathrm{nd}$ \\
Geranial & 1272 & 9,4 & nd & nd & nd \\
Formiato de citronelila & 1297 & 3,9 & nd & nd & nd \\
Formiato de geranila & 1439 & 5,0 & nd & nd & nd \\
6,9-Guaiadieno & 1479 & 1,2 & nd & nd & 4,0 \\
$\gamma$-Muuroleno & 1581 & nd & 13,4 & 3,3 & nd \\
\hline Óxido de cariofileno & & $\mathbf{8 3 , 4}$ & $\mathbf{9 5 , 6}$ & $\mathbf{9 2 , 5}$ & $\mathbf{9 4 , 8}$ \\
\hline Total & &
\end{tabular}

${ }^{a}$ Compostos listados em ordem de eluição na coluna ZB-5. bÍndice de retenção relativo de n-alcanos C9-C20 na coluna ZB-5. 'Percentuais obtidos pela normalização das áreas dos picos. ${ }^{\mathrm{d}}$ não detectado.

Apesar do óleo essencial de L. alba (LA-57), quimiotipo limoneno-carvona, apresentar limoneno $(36,0 \%)$ e carvona $(54,8 \%)$ como constituintes majoritários (Tabela 3), sua alta toxicidade deve estar associada principalmente à presença da carvona, pois como os resultados mostram que, na dose de $3 \mu \mathrm{g}$ do composto/mg de inseto, $84 \%$ das lagartas morreram após 24 h enquanto que apenas $4 \%$ morreram quando foi utilizado limoneno na mesma dose (Tabela 2). Entre alguns compostos terpênicos estudados por Abdelgaleil et al. (2009), ${ }^{21}$ a carvona foi o mais tóxico (via contato em superfície contaminada) para os coleópteros-praga de grãos armazenados Sitophilus oryzae (L.) e Tribolium castaneum (Herbst), com $\mathrm{CL}_{50}$ de 28,17 e $19,80 \mu \mathrm{g} / \mathrm{cm}^{2}$, respectivamente, o que comprova a ação inseticida deste composto.

O óleo essencial de L. alba, LA-10 (quimiotipo neral-geranial) é composto majoritariamente de neral $(32,9 \%)$ e geranial $(47,0 \%)$ (Tabela 3). Dessa forma, a toxicidade de citral (mistura isomérica cis-trans de neral e geranial) também foi avaliada, o qual causou mortalidade de $64 \%$ das lagartas na dose de $3 \mu \mathrm{g}$ do composto/mg de inseto (Tabela 2), comprovando que a alta toxicidade desse óleo está associada, principalmente, à ação sinérgica de neral e geranial.

A toxicidade do óleo essencial de L. alba (LA-22), quimiotipo linalol-1,8-cineol, foi semelhante aos demais óleos essenciais estudados, com base na comparação dos intervalos de confiança das doses letais estimadas. Apesar de o óleo essencial apresentar como constituintes majoritários o linalol $(81,0 \%)$ e 1,8-cineol $(8,2 \%)$ (Tabela 3$)$, sua toxicidade está relacionada principalmente à presença 
do composto linalol, visto que na dose de $3 \mu \mathrm{g} / \mathrm{mg}$ de inseto (correspondendo aproximadamente a $\mathrm{DL}_{90}$ estimada para o respectivo óleo essencial) houve mortalidade de $90 \%$ dos exemplares expostos e apenas $2 \%$ de mortalidade para 1,8-cineol na mesma dose, que não diferiu do controle (Tabela 2). Entre os três compostos terpênicos (estragol, linalol e sabineno) estudados por Wang et al. (2011), ${ }^{22}$ linalol foi o mais tóxico (via aplicação tópica) sobre o gorgulho-do-milho, Sitophilus zeamais Motsch, causando significativa mortalidade $\left(\mathrm{DL}_{50}=13,90 \mu \mathrm{g} /\right.$ adulto $)$ o que comprova a expressiva ação inseticida do linalol.

\section{CONCLUSÕES}

Com base nos resultados dos bioensaios realizados nas condições pré-estabelecidas, é possível concluir que os óleos essenciais de $P$. graveolens (PEL-001) e L. alba (acessos LA-10, LA-22, LA-57) apresentam atividade inseticida para lagartas de terceiro instar de $S$. frugiperda, com $\mathrm{DL}_{50}$ de 1,$13 ; 1,24 ; 1,56$ e $1,20 \mu \mathrm{g} / \mathrm{mg}$ de inseto e $\mathrm{DL}_{90}$ de 2,56; 2,60; 3,75 e 3,08 $\mu \mathrm{g} / \mathrm{mg}$ de inseto, respectivamente, contudo sem haver diferença entre estes com base na comparação dos intervalos de confiança das respectivas estimativas $(p \leq 0,05)$. Geraniol, linalol, carvona e citral, compostos majoritariamente presentes nos óleos essenciais estudados, ocasionam significativa mortalidade larval de $S$. frugiperda em comparação com o bioinseticida natural $\left(\right.$ Azamax $\left.{ }^{\circledR}\right)$ e com o controle negativo.

\section{AGRADECIMENTOS}

À CAPES e CNPq pelas bolsas concedidas e ao PROCAD 081/2007, CNPq e FAPESP pelos auxílios financeiros.

\section{REFERÊNCIAS}

1. Valicente, F. H.; Cruz, I.; EMBRAPA/CNPMS, Circular Técnica, n. 115, EMBRAPA: Sete Lagoas, 1991.

2. Cruz, I.; EMBRAPA-CNPMS, Circular Técnica, n. 21. EMBRAPA: Sete Lagoas, 1995.

3. Simões, C. M.; Spitzer, V. Em Farmacognosia: da planta ao medicamento; Simões, C. M. O.; Schenkel, E. P.; Gosmann, G.; Mello, J. C. P; Mentz, L. A.; Petrovick, P. R., eds.; $5^{\text {a }}$ ed., Editora UFRGS, Editora UFSC: Florianópolis, 2004.

4. Vale, T. G.; Matos, F. J. A.; Lima, T. C. M.; Viana, G. S. B.; J. Ethnopharmacol. 1999, 167, 127.

5. Henebelle, T.; Sahpaz, S.; Dermont, C.; Joseph, H.; Bailleul, F.; Chem. Biodivers. 2006, 3, 1116.
6. Gupta, R.; Sastry, K. P.; Banerjee, S.; Mallavarapu, G. R.; Kumar, S.; Genet. Resour. Crop Evol. 2001, 48, 629; Wüst, M.; Rexroth, A.; Beck, T.; Mosandl, A.; Flavour Frag. J. 1997, 12, 381.

7. Kulkarni, R. N.; Mallavarapu, G. R.; Baskaran, K.; Ramesh, S.; Kumar, S.; Flavour Frag. J. 1998, 13, 389; Lis-Balchin, M.; Steyrl, H.; Krenn, E.; Phytother. Res. 2003, 17, 60; Ram, P.; Kumar, B.; Naqvi, A. A.; Verma, R. S.; Patra, N. K.; Flavour Frag. J. 2005, 20, 666; Rana, V. S.; Juyal, J. P.; Blazquez, M. A.; Int. J. Aromather. 2002, 12, 216.

8. Agarwal, M.; Walia, S.; Dringra, S.; Khambay, B. P. S.; Pest Manag. Sci. 2001, 57, 289; Harborne, J. B.; Ecological biochemistry, $4^{\text {th }}$ ed., Academic Press: London, 1993; Isman, M. B.; Wan, A. J.; Passreiter, C. M.; Fitoterapia 2001, 72, 65; Lima, R. F.; Cardoso, M. C.; Santos, C. D.; Moraes, J. C.; Néri, D. K. P.; Nascimento, E. A.; Ciênc. Agrotec. 2009, 33, 1777; Prates, H. T.; Leite, R. V.; Craveiro, A. A.; Oliveira, A. B.; J. Braz. Chem. Soc. 1998, 9, 193; Tapondjou, A. L.; Adler, C.; Fontemc, D. A.; Bouda, H.; Reichmuth, C.; J. Stored Prod. Res. 2005, 41, 91.

9. Labinas, M. A.; Crocomo, W. B.; Acta Scientiarum (Agronomy) 2002, 24, 1401; Castro, D. P.; Cardoso, M. G.; Moraes, J. C.; Santos, N. M.; Baliza, D. P.; Rev. Bras. Plantas Med. 2006, 8, 27.

10. Paula, V. F.; Barbosa, L. C. A.; Demuner, A. J.; Piló-Veloso, D.; Picanço, M. C.; Pest Manag. Sci. 2000, 56, 168.

11. Kasten, P. Jr.; Precetti, A. A. C. M.; Parra, J. R. P.; Rev. Agric. 1978, 53, 68; Parra, J. R. P. Em Controle microbiano de insetos; S. B. Alves (Ed.). Manole: São Paulo, 1986.

12. Finney, D. J.; Probit analysis. Cambridge University Press: Cambridge, 1971.

13. Estrela, J. L. V.; Fazolin, M.; Catani, V.; Alécio, M. R.; Lima, M. S.; Pesq. Agropec. Bras. 2006, 41, 217.

14. Leora Software. Poloplus 1.0: probit and logit analysis. Berkeley, U.S.A. 2003. 1 CD-ROM.

15. Nelder, J. A.; Wedderburn, R. W. M.; J. R. Stat. Soc. 1972, 135, 370.

16. Demétrio, C. G. B.; Hinde, J.; GLIM Newsletter 1997, 27, 19; Hinde, J.; Demétrio, C. G. B.; Comput. Stat. Data Anal. 1998, 27, 151.

17. $\mathrm{R}$ Core Team. $R$ : A language and environment for statistical computing. R Foundation for Statistical Computing: Vienna, 2012.

18. Adams, R. P.; Identification of Essential Oil Components by Gas Chromatography/mass Spectroscopy, $4^{\text {th }}$ ed., Allured: Carol Stream, 2007.

19. Van den Dool, H.; Kratz, P. D. J.; J. Chromatogr. A 1963, 11, 463

20. Atkins, E.L.; Gerywood, E.; Macdonald, R.L.; Toxicity of pesticides and other agricultural chemicals to honey bees. Laboratory studies. University of California: Davis, 1976.

21. Abdelgaleil, S. A. M.; Mohamed, M. I. E.; Badawy, M. E. I.; El-Arami, S. A. A.; J. Chem. Ecol. 2009, 35, 518.

22. Wang, C. F.; Yang, K.; Zhang, H. M.; Cao, J.; Fang, R.; Liu, Z. L.; Du, S.; S.; Wang, Y, Y.; Deng, Z. W.; Zhou, L.; Molecules 2011, 16, 3077. 